\title{
Las exigencias de la calidad en la educación universitaria y la aplicabilidad de los derechos del consumidor: análisis actual y propuesta de mejora
}

\section{The requirements of quality in university education and the applicability of consumer rights: current analysis and proposal for improvement}

\author{
Diana Cecilia González Maldonado ${ }^{1 *}$, Segundo Germán Vélez Crespo ${ }^{1}$ y Froilán Segundo Méndez Velez ${ }^{1}$ \\ ${ }^{1}$ Universidad Católica de Cuenca \\ *dcgonzalezm@ucacue.edu.ec
}

DOI: https://doi.org/10.26871/killkana_social.v2i4.310

\begin{abstract}
Resumen
La escasa difusión de los derechos del consumidor en el ámbito de la educación superior genera preocupación, puesto que éstos, son el medio que permite garantizar el servicio a los estudiantes. El presente trabajo tiene como objeto demostrar que, la calidad de educación se ampara en la Ley Orgánica de Defensa del Consumidor y, no solo permite garantizar y hacer efectivos los derechos del consumidor, sino también contribuye con la misma en su carrera universitaria al recibir un título que sea reconocido y acreditado por la SENESCYT . Bajo este marco, se abordarán algunos aspectos teóricos relacionados a la Ley Orgánica de Defensa del Consumidor con sus respectivas responsabilidades y obligaciones de quien oferta un bien y/o servicio, en cumplimiento de la Ley Orgánica de Educación Superior, el Consejo de Aseguramiento de la calidad de la Educación Superior y el reglamento interno de la Universidad Católica de Cuenca (UCACUE) sede Azogues, por lo planteado, se realizará un estudio de los servicios de educación que ofrece la institución con énfasis en la calidad de la educación; entonces, se busca aplicar un análisis inductivo a partir de la situación actual de la prestación del servicio educativo, el cumplimiento de los parámetros de calidad y proponer una mejor aplicación de la Ley Orgánica del Consumidor, que favorezca al estudiante al momento de recibir el servicio educativo.
\end{abstract}

Palabras clave: Derechos, Consumidor, Educación Superior, Proveedor.

\begin{abstract}
The limited dissemination of consumer rights in the field of higher education raises concerns, since these are the means to guarantee service to students. The present work aims to demonstrate that the quality of education is covered by the Organic Law of Consumer Protection and, not only allows to guarantee and make effective the rights of the consumer, but also contributes with the same in his university career to receive a title that is recognized and accredited by SENESCYT. Under this framework, some theoretical aspects related to the Organic Law of Defense of the Consumer with their respective responsibilities and obligations of those who offer a good and / or service, in compliance with the Organic Law of Higher Education, the Board of Insurance of the quality of Higher Education and the internal regulations of the Catholic University of Cuenca (UCACUE) Azogues headquarters, so raised, a study of education services offered by the institution with emphasis on the quality of education will be conducted; then, it seeks to apply an inductive analysis from the current situation of the provision of educational service, compliance with quality parameters and propose a better application of the Organic Consumer Law, which favors the student at the time of receiving the service educational.
\end{abstract}

Key words: Rights, Consumer, Higher Education, Provider.

\section{Introducción}

La Ley Orgánica de Defensa del Consumidor en nuestro país, fue creada con el propósito de frenar todo tipo de abusos a los ciudadanos por parte de las empresas públicas o privadas de las que son consumidores o usuarios.
La Constitución ecuatoriana en su art. 52, manifiesta: "Las personas tienen derecho a disponer de bienes y servicios de óptima calidad y a elegirlos con libertad, así como a una información precisa y no engañosa sobre su contenido y características. La ley establecerá los mecanismos de 
control de la calidad y los procedimientos de defensa de las consumidoras y consumidores; y las sanciones por vulneración de éstos derechos, la reparación e indemnización por deficiencias, daños o mala calidad de bienes y servicios, y por la interrupción de los servicios públicos que no fuera ocasionada por caso fortuito o por fuerza mayor" (Asamblea Nacional, 2008).

La Ley Orgánica de Defensa del Consumidor se aplica con ciertas restricciones a los contratos de prestación de servicios educacionales, puesto que en su cuerpo normativo no precisa aquella materia especifica a las que se hacen extensibles las normas del consumidor. Mientras que el art. 353 de la Carta suprema del Estado determina que el Sistema de Educación Superior se regirá por un organismo público de planificación, regulación y coordinación interna del sistema y de la relación entre sus distintos actores con la Función Ejecutiva; y por un organismo público técnico de acreditación y aseguramiento de la calidad de instituciones, carreras y programas, que no podrá conformarse por representantes de las instituciones objeto de regulación. ( $L E Y$ ORGANICA DE EDUCACION SUPERIOR (LOES), 2010)

La educación superior en nuestro país asumió cambios fundamentales en los últimos años contando con un nuevo marco legal y regulatorio, situación que implica distintos requerimientos que cumplir en función de las expectativas generadas por las Universidades a nivel mundial, ya que educar es un acto que se refleja en la actuación del educando, por lo tanto se puede decir que la educación superior a influido sobre los miembros de sus comunidades con distintos fines, inclusive de índole productivo para asegurar la inserción laboral. De ahí que la educación superior es considerada como una herramienta de poder de algunos estados, puesto que los sectores dominantes la utilizan como un instrumento para mantener su hegemonía y se la considera como un bien público social; sin embargo a pesar de ello en los países llamados del tercer mundo la situación es diferente porque la educación no es un bien y hacen uso de ella sólo los grupos de poder para mantener las diferencias entre ricos y pobres.

La expansión de la oferta de educación superior, la diversidad y complejidad de carreras ofrecidas por instituciones prestadoras de servicios educacionales así como la noción de protección al consumidor han generado importantes cuestionamientos acerca de diferentes aspectos relacionados con la educación entre los cuales está su tratamiento como un bien de consumo a pesar de que en esta se involucran expectativas de desarrollo personal que son valoradas por los individuos y además comprometen una parte de su patrimonio.

Las nociones de calidad permiten comprender la vigencia de la misma en las instituciones de educación superior cuando determinan la forma de hacer las cosas en dichas organizaciones, por lo tanto la calidad es un conjunto de cualidades que posee una institución valoradas en tiempo y situación determinados que reflejan el modo de ser y actuar de la institución (Lemaitre \& López, 2016).
El mejoramiento de la calidad es imprescindible en la actualidad ya que conllevan la aptitud, el manejo de recursos adecuadamente y las acciones y esfuerzos que permitan alcanzar los propósitos de la institución.

Considerando las dimensiones política y pedagógica, es necesario replantear el derecho a la educación superior que poseen tanto hombres como mujeres ecuatorianos, refiriéndolo no solo al acceso sino también a las características de mejoramiento que permitan no sólo el éxito de los graduados sino su aporte como sujetos sociales al desarrollo cultural, socio - económico, político y ambiental del país, en base a ello es fundamental tomar en consideración la importancias que tiene la calidad de la educación superior en la participación de ,os actores sociales en los diferentes procesos de planificación, desarrollo de un determinado sector, asegurando de una mayor pertinencia en la oferta de servicios al generar una oferta educativa más significativa de acuerdo a las necesidades del mercado laboral (Consejo nacional de evaluacion y acreditacion. CONEA, 2003)

En la educación superior se considera consumidor al estudiante, mismo que cumplirá con ciertas obligaciones en el transcurso de su carrera universitaria, detalladas en los reglamentos de la institución educativa; mientras que el proveedor, es la entidad universitaria quien brindará los servicios de educación superior, siendo éstos de calidad. (Tobón, Rial, Carretero, y García, 2006)

Como señalara (Sánchez, 1995), "La educación superior formará profesionales capaces, que permita generar y conducir a cambios en la sociedad. Parte fundamental de ésta formación constituye la docencia universitaria que debe cumplir con las más altas exigencias de la Educación como lo establecen los organismos de control correspondientes" .

El Art. 89, de las Disposiciones Generales de la Ley Orgánica de Defensa del Consumidor, revela que el Ministerio de Educación y Cultura, incluirá en los planes de estudio un componente relacionado con la educación del consumidor, que permita hacer conocer a los estudiantes los derechos del consumidor (Ley orgánica de defensa del consumidor: porque todos los consumidores también tenemos derechos, 2010). Para las entidades de Educación Superior del Ecuador, el Consejo de Evaluación, Acreditación y Aseguramiento de la Calidad de la Educación Superior (CEAACES, 2015) , junto con el Consejo de Educación Superior (Consejo de Educación Superior, 2015), garantizarán la calidad y el mejoramiento de la educación superior, a través del cumplimiento de diversos indicadores.

En el presente trabajo se busca determinar si el servicio de la entidad educativa se lo hace con calidad, para ello se inicia definiendo al consumidor y al proveedor para luego realizar un análisis detallado de la situación actual de aplicación de los derechos del consumidor en relación a la calidad de la educación superior, su cumplimiento, haciendo referencia a ciertos artículos que garanticen el cumplimiento de la citada ley, puesto que la calidad de la educación superior debe aportar a los procesos de transformación social para llegar a ser una sociedad más pro- 
ductiva, equitativa, solidaria y justa considerando ciertos factores que influyen en la formación profesional, en la investigación, en la vinculación con la colectividad, en la administración y gestión universitaria.

\section{Metodología}

La educación superior ecuatoriana, se encuentra atravesando procesos de evaluación de su calidad académica, científica y tecnológica. No obstante, se pretende determinar la realidad de las universidades en relación a los derechos de los estudiantes como consumidor; entonces, el presente artículo se desarrolla mediante el uso del enfoque cualitativo, relacionando a lo largo del proceso varios métodos, a través del uso de información proporcionada por diferentes fuentes conocer como actualmente se encuentran empoderados los referidos derechos y el cumplimiento de la calidad en su oferta.

En efecto, el método deductivo se aplica, a través de la técnica de recopilación bibliográfica buscando establecer consideraciones generales de los temas de derechos del consumidor, calidad de la educación superior y evaluación, mismos que permitan cumplir con el objetivo plateado. Luego se utilizará el método descriptivo ya que a partir de los conceptos generales investigados se facilitará la comprensión de los servicios prestados por la institución educativa-proveedora. Por último se aplica el método inductivo, en virtud de que se realiza un análisis de la situación actual en el sistema educativo superior, proponiendo una mejora categórica del servicio, garantizando el cumplimiento de la normativa vigente y que le permita alcanzar los estándares de calificación establecidos; de esta manera, se llegará a conclusiones generales y principalmente a determinar la importancia que los actores del proceso de enseñanza-aprendizaje deben cumplir para mostrarse competitivos en su esfera de actuación.

\section{Resultados y Discusión}

\subsection{Consumidor: Derechos y obligaciones}

La Ley Orgánica de Defensa del Consumidor en nuestro país, promueve los derechos que tienen los ciudadanos cuando adquieren un bien o un servicio. Actualmente, pocos consumidores conocen esta ley, que permite hacer cumplir los derechos que les corresponden; por otra parte, las autoridades encargadas a defender al usuario, tienen la obligación de buscar los mecanismos que permitan dar efectivo cumplimiento a la norma legal, es más se conoce, que no dan la importancia que merece ante las denuncias recibidas por parte del consumidor.

En el Art. 2, del la presente Ley, se manifiesta que:" Consumidor es toda persona natural o jurídica que como destinatario final adquiera utilice o disfrute bienes o servicios, o bien reciba oferta para ello. Cuando la presente ley mencione al consumidor dicha denominación incluirá Usuario". (Ley orgánica de defensa del consumidor: porque todos los consumidores también tenemos derechos, 2010), por tanto, en el ámbito educativo, al estudiante se considera como el consumidor de la educación.

Dentro de los derechos y obligaciones del consumidor, están todos aquellos encaminados a proteger la vida al momento de consumir ciertos bienes o servicios para satisfacer las necesidades que se requieran; además "los consumidores tendrán derecho a que los proveedores públicos y privados oferten bienes y servicios competitivos de óptima calidad y a elegirlos con libertad". (Ley orgánica de defensa del consumidor: porque todos los consumidores también tenemos derechos, 2010), contemplados dentro del Art. 4, que refiriere a los derechos de los consumidores.

El estudiante universitario (consumidor), está en libertad de elegir la entidad educativa en la cual desee realizar sus estudios superiores, de contar con la información adecuada, clara, veraz y oportuna sobre los bienes y servicios ofrecidos, así como, el costo de los mismos, que permita recibir una educación universitaria adecuada para lograr una formación profesional eficaz (Aguilera Cabrera, 2005). El estudiante universitario, a través de la presente ley, está protegido de la publicidad engañosa o abusiva, que ofrezcan servicios desleales o coercitivos. Esta norma garantiza una difusión adecuada de la información por parte del proveedor.

En lo concerniente a las obligaciones del consumidor, el Art. 5 de la ley en análisis, manifiesta que el consumidor deberá realizar un consumo racional y responsable de los bienes y servicios que reciba, con el propósito de no poner en riesgo su vida y el medio ambiente, además de informarse de las condiciones y el uso de los mismos. (Ley orgánica de defensa del consumidor: porque todos los consumidores también tenemos derechos, 2010)

\subsection{Proveedor: responsabilidades y obligaciones}

La ley orgánica de Defensa del consumidor establece que los proveedores de bienes y servicios están en la obligación de proporcionar información necesaria (veraz, clara, completa, suficiente, etc.) para que éste pueda elegir de manera adecuada el bien o servicio que satisfaga sus necesidades; la mayoría de firmas y empresas proveedoras lanzan sus ofertas con el propósito de captar una gran cantidad de clientes sin considerar la calidad del producto; existen publicidades que a simple vista resultan atractivas para dar a conocer un producto, pero en algunas ocasiones la realidad es distinta cuando el servicio y el costo del producto varían radicalmente, así en la ley orgánica de defensa del consumidor las responsabilidades por parte del proveedor garantizaran en todo momento que el consumidor de bienes o servicios reciba un producto de calidad, con un precio justo y en caso de existir alguna falencia la reposición del mismo. (Brunner y Uribe, 2007)

Para el presente análisis el proveedor del servicio de educación se considera a la institución universitaria (Universidad Católica de Cuenca Sede Azogues) que en su visión manifiesta que se constituirá en una Casa de Estudios superiores defensora de los valores éticos y cristianos, 
además de ser debidamente acreditada, con excelencia en la docencia con el propósito contribuir al desarrollo de la sociedad y garantizar la calidad de educación impartida en sus aulas universitarias. (Universidad Católica de Cuenca, 2012)

En el art. 89 de la ley del consumidor manifiesta "que el Ministerio de Educación y Cultura incluirá en su pensum de estudios un componente relacionado a la educación del consumidor y que además la educación del consumidor privilegiará las siguientes áreas:

1) El conocimiento de derechos y obligaciones

2) Promover la capacidad para elegir con mayor libertad $\mathrm{y}$ eficacia entre los bienes y servicios que ofrece el mercado.

3) Planificar y satisfacer mejor sus necesidades; y,

4) Evitar riesgos derivados de un uso inadecuado de bienes y servicios" (Consumidor, 2011)

$\mathrm{El}$ art. 93 de la LOES manifiesta que el principio de calidad consiste en la búsqueda constante y sistemática de la excelencia, la pertinencia, producción óptima, transmisión del conocimiento y desarrollo del pensamiento mediante la autocrítica, la crítica externa y el mejoramiento permanente. (LEY ORGANICA DE EDUCACION SUPERIOR (LOES), 2010)

Y en concordancia con la Constitución de la Republica del Ecuador 2008, Arts. 346, la LOES en su art. 94. Hace referencia a la Evaluación de la Calidad y manifiesta que: es el proceso para determinar las condiciones de la institución, carrera o programa académico, mediante la recopilación sistemática de datos cuantitativos y cualitativos que permitan emitir un juicio o diagnóstico, analizando sus componentes, funciones, procesos, a fin de que sus resultados sirvan para reformar y mejorar el programa de estudios, carrera o institución. (LEY ORGANICA DE EDUCACION SUPERIOR (LOES), 2010)

En base a lo mencionado anteriormente la Universidad Católica de Cuenca garantizará su calidad educativa, al cumplir todas las disposiciones de los organismos de evaluación y acreditación (CES y CEAACES), ya que la evaluación institucional de universidades y escuelas politécnicas tiene como objetivo determinar el grado de cumplimiento de los estándares de calidad definidos en el modelo de evaluación vigente. (CEAACES, 2015)

\subsection{Análisis de la situación actual}

La educación superior juega un papel importante no solo en la formación de profesionales sino en la generación de nuevos conocimientos que permitan responder a las necesidades de la sociedad a lo largo del tiempo. Si se hace referencia a la sociedad del conocimiento, la formación profesional debe centrarse en el desarrollo de ciertas habilidades y en la resolución creativa de problemas imprevistos que puedan surgir en la actividad laboral, en este sentido la Universidad que es la entidad educativa que proporciona los servicios de educación superior al ser considerada como una persona de derecho privado sin fines de lucro se la consideraría de acuerdo al art. 564 "Se llama persona jurídica una persona ficticia, capaz de ejercer derechos y contraer obligaciones civiles, y de ser representada judicial y extrajudicialmente." (Congreso Nacional, s.f.)

Las personas jurídicas son de dos especies: corporaciones, y fundaciones de beneficencia pública. Hay personas jurídicas que participan de uno y otro carácter" (Congreso Nacional, s.f.), viene cumpliendo lo establecido en su visión e integra a todos los miembros participantes de la comunidad universitaria al impulso de las fuentes y medios de producción para brindar una educación de calidad basada en el Buen vivir, procurando ser una conciencia dinámica de las estructuras sociales basada en la comprensión, solidaridad, diálogo y respeto mutuo.

La Universidad Católica de Cuenca sede Azogues al encontrarse ubicada en un entorno con el cual interactúa y al estar presente en la sociedad azogueña, está condicionada por las características y tendencias del sistema social al que pertenece, los desarrollos políticos económicos y sociales imponen nuevos requerimientos a los egresados, quienes forman parte importante dentro de los cambios que se susciten en este contexto, lo que genera el incremento de la demanda a dicha institución y sobre todo en nuestro medio al ser la única universidad existente en la ciudad. El profundizar una cultura de calidad le permite a esta entidad direccionar su organización y establecer objetivos con bases sólidas y con miras a lograr mejoras en los procesos de docencia, investigación y vinculación; la evaluación de la calidad en una universidad requiere de antemano la determinación de sus propósitos u objetivos perseguidos, aunque hay que considerar que los objetivos son múltiples y que las valoraciones de ellos son diferentes. (Didou, 2005)

La calidad en la formación de los profesionales precisa de propuestas innovadoras, flexibles y dinámicas de acuerdo a las necesidades de la sociedad que permitan vincular la docencia, la investigación y la interacción social al formar individuos con valores éticos capaces de dar solución a las necesidades de la sociedad a través de aprendizajes significativos y críticos de manera que los estudios sean reconocidos en el ámbito nacional e internacional.

Para alcanzar los objetivos y sobre todo para el logro de la calidad en la educación se considera que: "La universidad actual se enfrenta al desafío de insertarse en un mundo complejo, con nuevas exigencias de profesionalismo y competencias emergentes asociadas a la sociedad del conocimiento. Se trata de construir una universidad que tenga buenas relaciones con la comunidad académica, que incorpore valor agregado a la experiencia de sus estudiantes, que disponga de los ambientes adecuados, que tenga un currículo apropiado, que implemente una investigación relevante, que realice una evaluación pertinente y que genere una gestión de la calidad de la educación superior". (González y Espinoza, 2008).

Considerando lo mencionado anteriormente en la actualidad la universidad Católica de Cuenca sede Azogues se encuentra inmersa en un proceso de acreditación cuyo 
eslogan manifiesta que:'La acreditación es responsabilidad de todos" (Universidad Católica de Cuenca, s.f.), y a través del cumplimiento de ciertos parámetros establecidos por la LOES Y la CEAACES; y, mediante la aplicación de un "Modelo para la Evaluación de Carreras con fines de acreditación" que servirá como instrumento orientador de los procesos de autoevaluación y evaluación se busca evidenciar que las actividades que se desarrollan en la universidad se encuentren en concordancia con la misión, visión, propósitos y objetivos institucionales para certificar ante la sociedad la calidad en la educación y la integridad institucional. Varios son los criterios de evaluación establecidos en el modelo, de entre los más importantes están: los objetivos educacionales, el currículo y los resultados de aprendizaje.

Para el cumplimiento de los criterios antes mencionados la entidad educativa en el ámbito académico cuenta con profesores capacitados en cada área estableciendo como requerimiento mínimo poseer maestría para ser docente, además se ha contratado profesores con el título de doctores o PHD que impartan conocimientos que garanticen la calidad de educación y fomenten la cultura de la investigación al estudiante en la aulas universitaria, también se ha incrementado en número de docentes a tiempo completo y medio tiemplo quienes cumplen actividades de docencia y otras como son la vinculación de la universidad con la comunidad, el desarrollo de proyectos de investigación con la participación y apoyo de los estudiantes, asesoría y otras que requiera la universidad; así como el apoyo mediante becas a los decentes para que realicen sus estudios de cuarto nivel. Garantizado de esta manera que la institución posea profesionales de elevada calidad profesional y humana con compromiso social en el diseño, planificación, ejecución y evaluación de los procesos curriculares como actividades que apoyan el cumplimiento de la Visión y Misión institucional.

El proceso formativo de los estudiantes permite una formación significativa que supera las debilidades en el proceso de ingreso de postulantes a la formación profesional, lo que posibilita el incremento de los índices de las tasas de retención y graduación, evitando así el desperdicio de recursos y aportando con un mayor número de profesionales a la sociedad, lo que permite cumplir además con los derecho del consumidor cuando adquiere los servicios de educación superior.

En la Universidad Católica de Cuenca Sede Azogues el proceso de enseñanza - aprendizaje desarrollado en base al pensamiento crítico y a la investigación formativa, permite que los alumnos desarrollen su capacidad para investigar, autoformarse de forma permanente y contribuyan creativamente a la solución de los problemas que les plantea su práctica profesional los que les permite dar solución a los problemas cotidianos; así mismo la realización de evaluaciones permanentes del desempeño a los docentes garantiza que se brinde una educación de calidad, aunque de acuerdo a los estándares establecidos por las organismos evaluadores de sebe seguir incrementando el porcentaje de docentes con títulos doctorado que permitan contribuir al conocimiento a través de la investigación científica.

En los que se refiere a la infraestructura la institución educativa cuenta con espacios físicos adecuados, laboratorios, acceso a la información, entre otros servicios que benefician directamente al consumidor de la educación al brindarle las condiciones adecuadas en le recepción del servicio educacional, sin embargo de acuerdo a las exigencias de los organismos evaluadores se debe propender a la acreditación de la sede universitaria de acuerdo a los criterios de evaluación establecidos por lo que la institución se encuentra implementado la infraestructura de acuerdo los requerimientos de la oferta académica

El modelo pedagógico por competencias basado en el "Aprender haciendo" hace que el estudiante sea parte activa del mismo y esté implicado directamente en su propio aprendizaje y una actitud crítica en el desempeño personal, social, político y cultural, sumando el apoyo del docente a través de actividades innovadoras que promuevan la práctica de los valores y coadyuven a la formación del ciudadano social y mejorando la interacción docente alumno; además se realiza un trabajo cooperativo por parte de los estudiante en las aulas universitarias y en el desarrollo de de proyectos y vinculación de la universidad con la comunidad con la finalidad de que se ponga en práctica los conocimientos adquiridos y el estudiante se desenvuelva en el campo profesional; actividades que garantizan que la educación impartida sea de calidad y este acorde a la realidad. "El modelo definido por el CEAACES tiene como principio guía colocar al estudiante como protagonista y al aprendizaje como el objetivo principal. Esto significa que se trata de valorar lo que el estudiante aprende efectivamente y no - como en una visión cronológicamente superada lo que el profesor enseña o lo que el estudiante debería conocer. Esta perspectiva se traducirá en el desarrollo de logros del aprendizaje que faciliten una efectiva movilidad de estudiantes y docentes a nivel nacional e internacional, no solamente en el ámbito académico, sino también en el profesional" (CEAACES, 2015)

Los procesos de investigación en la sede universitaria deben se ausmidos como una actividad institucional y deben estar orientados a la indagación, conocimiento de la realidad, su sistematización y su utilización para satisfacer las necesidades de la sociedad ya que en la actualidads estos porceso on todavía deficientes.y se requiere que la institución elabore programas de investugaciónrealcionados son ola consecusión de la Visión y Misión de la universidad y contribuyan al desarrollo de la misma y al de la sociedad de lorfa sosnteble.

La realización de actividades de vinculación le permiten a la universidad interactuar con otros actores de la sociedad al poner a disposición servicios especializados como asesorías, educación continua y consultorías que permitan contribuir a la solución de problemas y aportar 
al desarrollo local, regional y nacional, siendo fundamental el involucramiento de docentes, investigadores y alumnos.

Por lo tanto de acuerdo al art. 95 de la LOES, la Acreditación es una validación de vigencia quinquenal realizada por el Consejo de Evaluación, Acreditación y Aseguramiento de la Calidad de la Educación Superior, para certificar la calidad de las instituciones de educación superior, de una carrera o programa educativo, sobre la base de una evaluación previa. (LEY ORGANICA DE EDUCACION SUPERIOR (LOES), 2010). Siendo la acreditación producto de una evaluación rigurosa del cumplimiento de de lineamiento y estándares y criterios de calidad de nivel internacional, a las carreras y programas de educación, los cuales si se cumple de forma adecuada garantizarán que el consumidor de la educación superior adquiera un servicio de calidad.

El estudiante que se encuentra matriculado en la Universidad Católica de Cuenca sede Azogues, goza de varios derechos establecidos en el Estatuto y reglamento de la entidad de los cuales podemos enumerar los siguientes: integrar los organismos estudiantiles, obtener el correspondiente título profesional, derecho al reclamo, atención en sus peticiones y justificaciones, entre otros, que además de garantizar el bienestar estudiantil se cumplen a cabalidad por parte del estudiante ya que la entidad educativa ha implementado un buzón de sugerencias mediante el cual el departamento de Bienestar Estudiantil se encarga de solucionar los problemas y considerar las sugerencias que realizan los estudiantes. (Universidad Católica de Cuenca, s.f.)

El Art. 19 de la Ley Orgánica de Defensa del Consumidor manifiesta:" Indicación del precio. - Los proveedores deberán dar conocimiento al público de los valores finales de los bienes que expendan o de los servicios que ofrezcan, con excepción de los que por sus características deban regularse convencionalmente. El valor final deberá indicarse de un modo claramente visible que permita al consumidor, de manera efectiva, el ejercicio de sus derechos a elección, antes de formalizar o perfeccionar el acto de consumo..." (Ley orgánica de defensa del consumidor: porque todos los consumidores también tenemos derechos, 2010) disposición que se cumple a cabalidad por parte de la universidad ya que los estudiantes conocen el precio que deben pagar por las colegiaturas del año que van a cursar. Además de los derechos que garantizan la calidad de educación y el cumplimiento de los derechos del consumidor, el estudiante tiene ciertas obligaciones a cumplir.

Para cumplir con el objetivo de brindar una educación de calidad, las instituciones de educación superior requieren planificar el desarrollo institucional de manera estratégica y evaluar constantemente sus actividades para alcanzar el objetivo deseado CONEA, 2003), de ahí que el plan estratégico institucional de la UCACUE considera todas las actividades optimizando sus recursos en relación a la consecución de la Visión y Misión y a la vez que es una herramienta de gestión que implica la participación de los sectores internos y de la sociedad para lograr una mayor contribución al desarrollo de la misma y sobre todo para alcanzar la categoría A.

\subsection{Propuesta de mejora}

Luego de realizar el análisis de la Ley Orgánica de Defensa del Consumidor y relacionarla con el ámbito educativo de la Universidad Católica de Cuenca sede Azogues, la propuesta de mejora estaría dirigida al cumplimiento de los lineamientos y criterios establecidos por el Consejo de Educación Superior (CES) y Consejo de Evaluación, Acreditación y Aseguramiento de la Calidad de la Educación Superior (CEAACES) para garantizar la calidad de la educación.

En lo referente a la docencia universitaria se deberá capacitar a los docentes de forma permanente para aplicar el concepto de calidad como respuesta a los requerimientos del medio, basada en definiciones donde prima la pertinencia; además, corresponderá a la universidad incentivar a los docentes el desarrollo y ejecución de proyectos encaminados a buscar el bienestar de la sociedad junto con la colaboración de directivos y estudiantes; de igual manera se deberá seguir apoyando a los docentes a través de becas de estudios para que obtengan títulos de cuarto nivel y contratar más docentes con título de PHD para garantizar que los conocimientos impartidos en la aulas universitarias sean los mejores; y de esta manera asegurar que en el proceso formativo los estudiantes desarrollen su capacidad investigativa y al mismo la institución promueva procesos de investigación científica entre: universidades, centros de investigación, con el gobierno local y nacional y el sector productivo que beneficie al desarrollo de los diferentes sectores en los que el profesional de la UCAUE se desempeñe, para satisfacer las necesidades el consumidor de los servicios de educación.

Impulsar la investigación de forma sistemática y planificada articulada a la docencia, investigación y vinculación con la comunidad se logrará a partir del establecimiento de alianzas estratégicas con los sectores productivos, organismos de desarrollo gubernamental, no gubernamental, etc. con el propósito de responder a los requerimientos de la sociedad, con la participación del recurso humano calificado que proporcionaría el profesional de la UCACUE; para alcanzar el más alto nivel en la investigación, es necesaria la asignación de recursos económicos que propicien la excelencia científica y que sus resultados beneficien al entorno socio - económico y cultural y así mismo la universidad deberá publicar de forma permanente dichos resultados para difundir el avance del conocimiento a nivel institucional y dar a conocer el beneficio que recibe la sociedad lo cual le garantizará alcanzar los mejores estándares en educación y de esta manera también garantizar que se cumpla con la Ley Orgánica de Defensa del Consumidor.

La Universidad Católica de Cuenca, deberá ofertar nuevas carreras de acuerdo a las exigencias de la sociedad lo que permitirá al estudiante (consumidor) tener un abani- 
co de opciones al elegir la profesión que deseen y evitar la saturación de carreras existentes en el medio y sobre todo permitan la inserción de graduado en el campo laboral. A la universidad le corresponderá realizar firmas de convenios interinstitucionales tanto nacionales como extranjeros que permitan ofertar títulos de cuarto nivel para mejorar de forma continua la eficiencia y eficacia de la calidad de educación.

Respetar el sistema jurídico por parte de la universidad se reflejará en los procesos democráticos ya que el respeto a la Constitución de la República, leyes generales y especiales, reglamentos y otras normas legales le permitirán a la institución fortalecerse al poner el práctica de manera estricta la normativa existente ya será al interior o al exterior de la misma o en la relaciones que mantiene con la comunidad, lo que fortalecerá la imagen institucional ya que los servicios que ofrece son los mismos que proporciona lo que garantiza el consumidor o estudiante recibe un servicio que satisfaga sus necesidades.

\section{Conclusiones}

Mediante el sondeo relacionado con la calidad del servicio ofertado por las Universidades a nivel nacional, se concluye:

- Para efectos de interpretación de la Ley Orgánica de Defensa del Consumidor se aplicará de forma favorable para el consumidor, es claro que no se garantice el cumplimiento de la misma puesto que los proveedores de bienes o servicios en la mayoría de ocasiones no cumplen con sus obligaciones.

- Del análisis realizado podemos concluir que se deben respetar y hacer cumplir a cabalidad los derechos de los consumidores y usuarios, y que estos derechos no deben ser violados por ninguna persona.

- En el ámbito educativo las decisiones que se tomen para promover la mejora continúa de la eficiencia y eficacia de las organizaciones educativas deben basarse en el análisis de la información y datos, así como de la adecuada interpretación de las normas de calidad, que garanticen proveer servicios de calidad al estudiante.

- El personal de la universidad en todos sus niveles debe estar involucrado en el desarrollo de su sistema de gestión de la calidad y adquirir el compromiso de que aporten experiencia y habilidades para que sean aprovechadas en beneficio de la organización y se logre el cumplimiento de los objetivos al ofertar servicios de calidad al consumidor (estudiante).

- La mejora continua de las organizaciones educativas contando con la participación y el consenso de directivos, docentes, estudiantes y de todas las partes interesadas se logra a través de acciones pertinentes para mejorar la calidad de educación. La mejora continua del desempeño global de las instituciones de educación superior debe ser un objetivo permanente de la gestión de la calidad educativa para ponerla a disposición del estudiante y garantizar el cumplimiento de la Ley orgánica de Defensa del Consumidor.

\section{Referencias Bibliográficas}

Aguilera Cabrera, V. (2005). El concepto de la calidad en la educación universitaria: Clave para el logro de la competitividad institucional. Revista Iberoamericana de Eduación, 2-8.

Asamblea Nacional. (2008). Constitucion de la republica del ecuador 2008.

Brunner, J. J., y Uribe, D. (2007). Mercados universitarios: el nuevo escenario de la educación superior. Chile: Ediciones: Universidad Diego Portales.

CEAACES. (2015). Descargado de http:// www. caces.gob.ec/web/ceaaces/ institucional

Congreso Nacional. (s.f.). Ministerio de Justicia. Descargado 22 de Mayo de 2012, de http:// www · justicia.gob.ec/wp-content/ uploads/2018/02/08-Codigo-Civil .pdf

Consejo de Educación Superior. (2015). Descargado de http://www.ces.gob.ec/doc/ Reglamentos_Expedidos_CES/ codificacin $\% 20$ del $\% 20$ reglamento\% 20 de $\% 20$ rgimen 20 acadmico.pdf

Consejo nacional de evaluacion y acreditacion. CONEA. (2003). La calidad en la universidad ecuatoriana. principios, características y estándares de calidad. Quito, Ecuador: IESALC.

Didou, S. (2005). Internacionalización y proveedores externos de educación superior en américa y el caribe. México D.F: ANUIES.

González, L., y Espinoza, O. (2008). Calidad en la educación superior: Conceptos y modelos. la profesión académica. Descargado de https://dialnet.unirioja.es/ servlet/articulo? codigo=3215107

Ley organica de educacion superior (loes). (2010). Descargado 12 de octubre de 2010, de http://www.yachay.gob.ec/ wp-content/uploads/downloads / 2013/12/LEY-ORGANICA-DE-EDUCACION -SUPERIOR-ANEXO-a_1_2.pdf

Ley orgánica de defensa del consumidor: porque todos los consumidores también tenemos derechos (1. ed ed.). (2010). Quito, Ecuador: Tribuna Ecuatoriana de Consumidores y Usuarios [u.a.]. (OCLC: 837751284)

Sánchez, D. (1995). Modelos académicos. Texas: Asociación Nacional de Universidades e Instituciones de Educación Superior.

Tobón, S., Rial, A., Carretero, M., y García, J. (2006). Competencias, calidad y educación superior. Bogotá: Cooperativa Editorial Magisterio. 
Universidad Católica de Cuenca. (s.f.). Descargado Noviembre de 2013, de http: / / www . ucacue. edu . ec/Acreditacion.aspx

Universidad Católica de Cuenca. (2012). Vademécum legal исасие. Descargado de https:// documentacion.ucacue.edu.ec/items/ browse

Recibido: 30 de julio de 2018 Aceptado: 7 de septiembre de 2018 